\title{
MODELING OF THE COMETARY NUCLEUS-COMA INTERFACE REGION
}

\author{
T. I. Gombosi* and A. Körösmezey** \\ * Space Physics Research Laboratory, Department of \\ Atmospheric, Oceanic and Space Sciences, The University of Michigan, \\ Ann Arbor, Michigan 48109, U.S.A. \\ * Space Physics Research Laboratory, Department of \\ Atmospheric, Oceanic and Space Sciences, The University of Michigan, \\ Ann Arbor, Michigan 48109, U.S.A. and Central \\ Research Institute for Physics, Hungarian Academy of Sciences, Budapest \\ P.O. Box 49, Hungary 1525
}

\section{ABSTRACT}

A well-developed dusty cometary atmosphere extends to distances over 4 orders of magnitude larger than the size of the nucleus. Pre-encounter models of the inner coma were based on the assumption that a spherically symmetric description was adequate to describe the dust-gas interaction region. Recent observational evidence together with a new generation of multidimensional theoretical models demonstrate that the inner cometary environment is far from spherical symmetry and a number of unexpected phenomena (dust jet broadening, subsolar dust spike formation, etc.) might play a significant role in this region.

\section{INTRODUCTION}

Our present, post-encounter understanding of cometary nuclei is based on Whipple's "dirty iceball" idea /1/, which visualizes them as chunks of ice, rock, and dust with negligible surface gravity. Whipple's hypothesis quickly replaced the century-long series of "sandbank" models, wherein the nucleus was thought of as a diffuse cloud of small particles traveling together. As comets approach the sun, water vapor and other volatile gases sublimate from the surface layers generating a rapidly expanding dusty atmosphere. The sublimated gas molecules (often called parent molecules) undergo collisions and various fast photochemical processes in the near nucleus region, thus producing a whole chain of daughter atoms and molecules. There is growing evidence that delayed gas emission from dust particles, dust grain photochemistry, as well as gas - dust chemical reactions also contribute to the maintenance of cometary atmospheres.

In the vicinity of the nucleus the gas and dust flows are strongly coupled: frequent gas - dust collisions accelerate small grains to velocities up to several hundreds of meters per second and inject them into the extensive cometary exosphere, where the gas and dust are decoupled. The expanding gas eventually converts most of its original internal energy to bulk motion, while it also loses momentum and energy to the dust flow. At the same time the nucleus surface and the accelerating dust grains are heated by the attenuated and multiply scattered solar radiation. Most of the thermal radiation of the solid components is emitted in the $1-20 \mu \mathrm{m}$ wavelength range, where several rotational and vibrational transients exist for the highly dipolar water molecules which have very large resonance cross sections. At the resonant frequencies the radiation is continuously absorbed and reemitted by the water molecules: in other words it is trapped by the gas $2 \%$. A large fraction of the rotational/vibrational excitation energy is transformed into translational motion via molecular collisions, thus increasing the gas temperature. The higher gas temperature represents an increased source of internal energy, which eventually results in higher terminal velocities due to adiabatic cooling.

One of the most important factors influencing cometary dynamics is the "retarded" nature of gas and dust production. The radiation reaching the surface and supplying energy for sublimation must first penetrate an extensive, absorbing dusty atmosphere. Any change in the gas and dust production alters the optical characteristics of the atmosphere, thus causing a delayed (or "retarded") effect on the production rates themselves. This "retardation" makes inner coma modeling efforts complicated and time consuming.

It was recognized as early as the mid-1930s that gas outflow plays an important role in cometary dust production. In early treatments of the gas-dust interaction it was assumed that the gas drag coefficient was independent of the gas parameters and that the gas velocity was constant in the dust acceleration region. In the late 1960 s this very naive picture was replaced by a two component approach, which used free molecular approximation to describe the rarefied gas flow and neglected the random motion of the dust particles. In Probstein's 3 / dusty gas dynamic treatment (which later became the prototype of such calculations), the traditional gas energy conservation equation was replaced by a combined dust-gas energy integral. This approach was later considerably refined by a series of authors $/ 2 /, 14-$ $12 /$, but it still represents the main method of dusty gasdynamics calculations.

Modeling efforts have shown that the spatial extent of the dust acceleration region (where dust particles accelerate to about $80 \%$ of their terminal velocity) is less than about 30 cometary radii. Gas particles typically spend less than $10^{2}$ seconds in this region, which is not long enough for any significant change in the gross chemical composition of the gas. In a first approximation a single-fluid dusty gas hydrodynamical technique seems to be adequate for describing the overall dynamics of the gas-dust interaction. 
Spherically symmetric steady-state approximation of the dusty gas mixture system yieids a solar-wind type equation, which describes a transonic flow in the immediate vicinity of the nucleus. The equation has a $0 / 0$ type singularity at the sonic point, causing many numerical complications. Earlier models assumed a supersonic gas flow at large cometocentric distances and a subsonic flow close to the nucleus; in this case there is one and only one transonic solution which passes smoothly through the sonic point. Transonic solutions can not, in principle, be obtained numerically without additional assumptions. Generally, it has been assumed that the gas velocity (or Mach number) and its first derivative behaved continuously at the sonic point. Following Probstein's original work $/ 3 /$, practically all early transonic coma calculations used a type of "shooting method": the initial flow velocity (or Mach number) value was "fine tuned" until a transonic solution was reached $/ 6,8,9,10,13 /$. In order to avoid the numerical difficulties of the earlier treatments and also to make it possible to describe dynamic phenomena in the coma, the first time-dependent (but still spherically symmetric) dusty gas dynamic model of the gas-dust interaction region was developed by Gombosi et al. $\Pi /$. The method was later combined with a three-dimensional, kinetic dust treatment in the outer coma /14/. In this model the gas dynamic equations are solved using a modified version of Godunov's first scheme which can naturally handle shocks and discontinuity surfaces. The model was able to describe such phenomena as dust halo formation in the inner coma, temporal evolution of dust and gas parameters following a comet outburst, etc. However, the numerical model can still use further improvements. In its first published form the model assumed a rather simple photochemistry, gas collisional cooling, neglected infrared radiation trapping $2 /$, and assumed that the total radiative energy flux was approximately constant everywhere in the coma (an assumption that is more or less justified on the basis of earlier radiative transfer calculations /10,15/.

The comet Halley imaging experiments showed that cometary activity is concentrated to limited areas on the sunlit side of the nucleus, with most of the dust ejection coming from fairly localized jets. Spherically symmetric, steady-state models were proven to be totally inadequate to describe cometary inner regions. A couple of years ago Kitamura /11/ has developed the first time-dependent 2D dusty gas-dynamic code using one characteristic dust size, simple energetics, and a highly simplified chemistry. In a follow-up paper Kitamura $/ 12 /$ also investigated the multidimensional dynamics of isolated jets (when gas and dust is produced only by a limited active region). Kitamura's numerical technique $/ 11,12 /$ has problems with the time-dependent treatment of the gas outflow, therefore, he published only steady-state results. Also, his isolated jet model does not extend beyond the terminator. This limitation is important, because as it will be demonstrated later in this paper, strong lateral transport of dust particles might produce secondary dust density increases in the nightside of the comet. There is strong observational indication that such dust accumaltion was observed by the Giotto imaging experiment $/ 16 \%$. Nevertheless, his first $2 \mathrm{D}$ calculation represent an important step forward in describing dusty jets. Subsequently, Kömle and Ip /17/ published a two-dimensional time-dependent pure gasdynamic model (no dust was included) to study the evolution and interaction of isolated cometary gas jets. Recently Gombosi and Körösmezey /18/published a two-dimensional, truly time-dependent dusty gasdynamic model describing the evolution of localized cometary jets. Table 1 summarizes the main features of the present multidimensional cometary jet models.

TABLE 1 Comparison of Axisymmetric Jet Models

\begin{tabular}{|c|c|c|c|}
\hline KJtamura /IT & Kóme \& Ip /I7/ & Kitamura /127 & Gombosi \& Korösmezey /18] \\
\hline Dusty gas jet & Pure gas jet & Dusty gas jet & Dusty gas jet \\
\hline 1 dust size & No dust & 3 dust sizes & 1 dust size \\
\hline $\begin{array}{l}\text { Spherically symmetric } \\
\text { background emission }\end{array}$ & No background emission & No background emission & $\begin{array}{l}\text { Axisymmetric background } \\
\text { emission }\end{array}$ \\
\hline Subsolar jet & Subsolar jet, ring & Subsolar jet & Subsolar jet \\
\hline Steady-state results & Time-dependent resultis & Steady-state results & Time dependent results \\
\hline Day \& night flow & Day \& night tlow & Day flow only & Day and night flow \\
\hline
\end{tabular}

In the early dusty gas dynamic calculations, attenuation of the solar radiation field by dust grains and gas particles was neglected. Based on a spherically symmetric dust density distribution produced by an isothermal nucleus, Hellmich /13/ developed a model to calculate the transfer of multiply scattered radiation in the inner coma and to determine the energy input to the nucleus. Surprisingly, Hellmich $/ 13 /$ and later Weissman and Kieffer $/ 19 /$ found that the net effect of the dust on the sublimation somewhat enhances the gas and dust production because the radiative flux scattered within the inner dust coma is partially trapped and this effect over-compensates the attenuation of direct solar radiation. Marconi and Mendis $/ 9 /$ published an alternative model, which considered mainly the transfer of the solar UV radiation responsible for the major photolytic processes and treated the longer wavelength diffuse radiation field only superficially. It was also pointed out by Marconi and Mendis $/ 2 /$ that several rotational and vibrational transitions exist for the highly dipolar water molecular in the $1-20 \mu \mathrm{m}$ wavelength range, where most of the dust thermal radiation is emitted. In a collisionless gas the resonant radiation is continuously absorbed and reemitted by the water molecules: in other words it is trapped by the gas. On the other hand, collisions play an important role in the inner coma and a large fraction of the rotational/vibrational excitation energy of water molecules can be transformed via collisions into translational energy, thus increasing the gas temperature. Marconi and Mendis $/ 2 /$ have included a new approximate gas heating rate describing the effects of the dust thermal radiation and obtained a significant increase in the gas temperature and velocity profiles. The details of this complicated interaction process are not adequately understood, and the application of generalized transport equations may help us to have a better insight into this significant effect. 


\section{DUST PRODUCTION}

The chemical composition and physical structure of the surface layers of a cometary nucleus are very important factors affecting the mass, momentum and energy of the outflowing gas-dust mixture, as well as the relative abundances of various gas molecules. When the comet approaches the sun, it absorbs an increasingly larger flux of solar radiation, and the vaporization rate of volatile molecules at the surface increases. Gravitational forces are very small, therefore the vaporized gases leave the surface and form an expanding atmosphere. In this process the gas drags away some of those dust grains which have already been evacuated of their ice component (at least partially), but others may remain on the surface (or may fall back). In his original presentation of the icy-conglomerate model Whipple predicted that an inert layer of large dust particles, evacuated of the volatile component, would form an insulating crust on the surface (mantle) $/ 1 \%$. The thickness of the mantle varies with time because the continuous vaporization increases the thickness of the evacuated layer, and the "erosion" due to the drag of the outflowing gas decreases it. The development and thermal structure of such a mantle has been extensively discussed in the literature $14,20-24 /$. These models were able to predict several different mantle evolution patterns (for a detailed review refer to $/ 25 /$ ). The preencounter view of the mantle evolution process assumed that active periodic comets were covered with friable surface dust layers, so that a repetitive cycle appeared as the comet orbited the sun. The prevailing view was that apart from the first approach to the vicinity of the sun, the mantle thickness and the total gas production rate basically followed similar curves during subsequent revolutions. As a Halley-type comet approaches the sun the mantle thickness increases up to a critical heliocentric distance, and then it starts to decrease. By the time the comet passes its perihelion most of the mantle is blown off and it keeps eroding further resulting in additional postperihelion brightening. When the comet again leaves the vicinity of the sun, a new mantle is developed; this new mantle is blown off during the next perihelion passage.

Elementary gaskinetic theory has been used to calculate the gas production rate since the early work of Delsemme and Swings $/ 26 \%$. It was widely assumed that the sublimated gas molecules leave the vicinity of the nucleus without collisions, so that the outflow velocity could be approximated reasonably well by the local sound velocity. At the same time the typical mean free path of the gas molecules near a cometary nucleus is on the order of $10 \mathrm{~cm}-10 \mathrm{~m}$, therefore, hydrodynamic rather than kinetic approach is needed to calculate gas outflow rates. To resolve this problem, Gombosi et al. $/ /$ introduced a "reservoir outflow" model for gas production from the nucleus where the sublimating surface was replaced by a gas reservoir containing a stationary perfect gas. The surface of the reservoir was assumed to be covered by a thin layer of friable dust so that the gas could flow through it and which also "loaded" the discharging gas flow with dust grains. It was also assumed that the gas slowly diffused through the porous mantle and at every point was heated to the local mantle temperature. In this model the practically stationary gas at the top of the nucleus had a temperature identical to the mantle surface temperature and a pressure which was the same as the sublimation pressure. Gombosi et al. $\Pi /$ have considered the time dependent dusty gas outflow from such a nucleus, assuming a realistic surface and sublimating temperatures.

The comet Halley images revealed that most of the dust production (and supposedly the gas production, too) was concentrated on several active areas on the sunlit side, which covered only about $10 \%$ of the cometary surface. Careful analyses of ground based observations also helped to identify active spots and line sources, which turn on and off fairly randomly. All this evidence points toward a much more complex picture of cometary gas and dust production than described by our present models: it seems to be increasingly probable that thermal stresses and other effects can cause rapidly developing openings (cracks) in the surface layers, which later slowly "heal" as a new dust mantle develops. Presently there are only a few initial attempts to model such localized, random active areas (cf. 27/).

\section{COMA DYNAMICS}

As the vaporized gases leave the surface they drag away some of those dust grains which have atready been evacuated of their ice component. The gas drag force accelerates the dust particles to terminal velocities comparable with the gas flow velocity. The mass, momentum and energy conservation equations of the single-fluid neutral gas are the following:

$$
\begin{gathered}
\frac{D \rho}{D}+\rho \nabla \cdot \mathbf{u}=0 \\
\rho \frac{D u}{D}+\nabla p-\rho g_{c}=F_{g d} \\
\frac{1}{\gamma-1} \frac{D \rho}{D t}+\frac{\gamma}{\gamma-1} p \nabla \cdot \mathbf{u}=Q_{e x t}-Q_{g d}
\end{gathered}
$$

where

$$
\frac{\mathrm{D}}{\mathrm{D}}=\frac{\partial}{\partial t}+(\mathbf{u} \cdot \nabla)
$$

is the convective derivative, $\rho=$ mass density, $p=$ gas pressure, $u=$ gas velocity, $g_{c}=$ acceleration due to the gravitational attraction of the comet, $F_{\text {gd }}=$ gas to dust momentum transfer rate, $\mathrm{Q}_{\mathrm{gd}}=$ gas to dust energy transfer rate, $Q_{\text {ext }}=$ external heating rate of the gas. In the innermost coma where most of the gas-dust interaction takes place the radiation pressure effect can be neglected and the equation of motion of an individual dust grain with characteristic

$$
\frac{D V_{a}}{D t}=\frac{3}{4 a \rho_{a}} p C_{D} s_{a}+g_{c}
$$


where $v_{\mathbf{a}}=$ dust particle velocity. The dimensionless gas-dust relative velocity, $s_{\mathbf{a}}$, and the modified free molecular drag coefficient, $C D$, are $B /$ :

$$
\begin{gathered}
s_{a}=\frac{u-V_{a}}{\sqrt{2 \frac{k_{m}}{m}}} \\
C_{D}=\frac{2 \sqrt{\pi}}{3} \sqrt{\frac{T_{a}}{T}}+\frac{2 s_{2}^{2}+1}{s_{2}^{2} \sqrt{\pi}} e^{-s_{2}^{2}}+\frac{4 s_{1}^{4}+4 s_{2}^{2}-1}{2 s_{2}^{3}} \operatorname{erf}\left(s_{2}\right)
\end{gathered}
$$

where $s_{a}=$ magnitude of the dimensionless gas-dust relative velocity vector, while $T$ and $T_{a}$ are the gas and dust temperatures, respectively. In the presence of an external radiation field the energy balance equation for a single dust

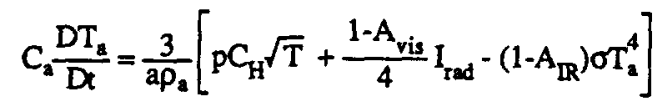

where $C_{a}=$ dust specific heat, $A_{\text {vis }}$ and $A_{I R}$ are the visible and infrared dust albedos, respectively, while

where

$$
C_{H}=\frac{\Gamma_{2}}{\gamma-1} \sqrt{2 \frac{k}{m}}\left[2 \gamma+2(\gamma-1) s_{a}^{2}-\frac{(\gamma-1) \operatorname{erf}\left(s_{a}\right)}{s_{a} \Gamma_{a}}-(\gamma-1) \frac{T_{a}}{T}\right]
$$

$$
\Gamma_{\mathrm{a}}=\sqrt{\pi} \mathrm{e}^{-s_{\mathrm{s}}^{2}}+\left(\frac{1}{2 s_{\mathrm{a}}}+s_{\mathrm{a}}\right) \operatorname{erf}\left(s_{\mathrm{a}}\right)
$$

Finally, it is assumed that the dust particles do not undergo any further sublimation or fragmentation in the coma (however, there is recent indication that this assumption is probably violated), consequently the dust size distribution function, $f_{a}$, must obey the following continuity equation:

$$
\frac{D f_{a}}{D}+f_{a} \nabla \cdot V_{a}=0
$$

The gas to dust momentum and energy transfer rates can be obtained by integrating over all dust sizes:

$$
\begin{gathered}
F_{g d}=2 \pi p \int_{0}^{2 \max } d a C_{D} a f_{a} s_{2} \\
Q_{g d}=2 \pi p \int_{0}^{a_{\max }} d a a f_{a}\left(C_{D} \nabla \cdot s_{a}+4 C_{H} \sqrt{T}\right)
\end{gathered}
$$

It should be noted that these integrals are dominated by the momentum and energy transfer to small particles. External gas heating is mainly caused by photochemical and radiative heating/cooling processes (cf. $25 /$ ), Q ext $=Q_{\text {phc }}+Q_{\text {IR }}$. The main contribution to the photochemical heating rate comes from the photodissociation of water molecules (cf $\Omega 28$ )

$$
Q_{\text {phe }}=Q_{0} \frac{n}{d^{2}} e^{-t_{w r}}
$$

where $\mathrm{n}=\rho / \mathrm{m}, \mathrm{Q}_{0}=2.8 \times 10^{-17} \mathrm{erg} \mathrm{cm} \mathrm{cm}^{-3} \mathrm{~s}^{-1}, \mathrm{~d}=$ heliocentric distance (AU), $\tau \mathrm{UV}=$ ultraviolet optical depth. The two main processes contributing to the infrared radiative heating/cooling term are the infrared radiation from the $\mathrm{H}_{2} \mathrm{O}$ molecules 29, 30/ and the radiative trapping of the dust thermal radiation Marconi and Mendis 2/.

Using the simplest, spherically symmetric steady-state approximation of the dusty gas mixture system yields a solarwind type equation, which describes a transonic flow in the immediate vicinity of the nucleus:

$$
\frac{d u}{d r}=-\frac{u}{1-M^{2}}\left(\frac{2}{r}-\frac{F_{g d}}{p}+\frac{(\gamma-1)\left(Q_{g d}-Q_{e x t}\right)}{\gamma p u}\right)
$$

Here $\mathrm{I}$ denotes cometocentric distance, while $\mathrm{M}$ is the flow Mach number. The physical solution describes a reservoir outflow to a low pressure external medium (the external pressure is at least ten orders of magnitude smaller than the pressure at the nucleus). This means that at large cometocentric distances the gas pressure must vanish while the flow velocity remains finite, i.e.,

$$
\lim _{r \rightarrow \infty} M=\infty
$$


The dust loading acts as a momentum sink for the gas component, consequently the outflow is subsonic (in reservoir outflow problems unobstructed flows leave the reservoir with the local sound velocity, mass loaded flows always have subsolar outflow velocities):

$$
M\left(r=R_{n}\right)<1
$$

Equations (16) and (17) mean that the only physical solution to equation (17) is a transonic flow.

It is interesting to note that equation (15) is quite analogous to the classic solar wind equation. In the cometary case the gas to dust momentum transfer replaces the effect of solar gravity, otherwise the mathematical form of the equation is essentially unchanged. Fig. 1 shows the various types of mathematically possible solutions, which are the same as the solutions of the solar wind equation.

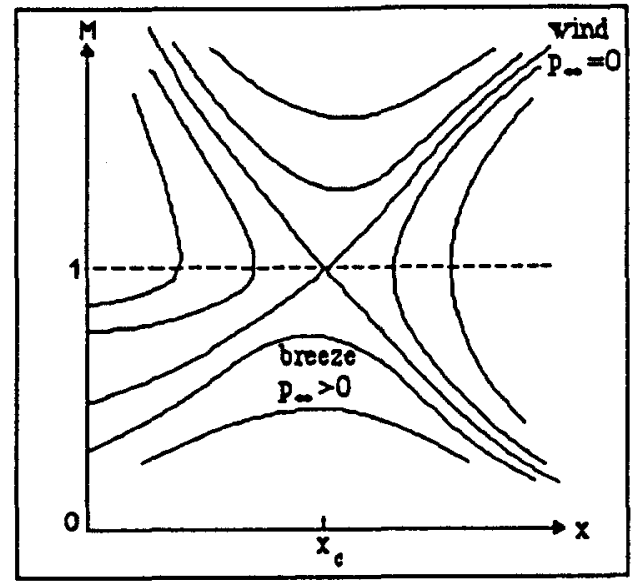

Fig. 1. Various types of solutions to equation (15). The only physical solution is the cometary wind, which starts subsonically at the surface, goes through a sonic point and monotonously increases in the supersonic region.

The physical solution of equation (15) has a $0 / 0$ type singularity at the sonic point, causing many numerical complications. Earlier models assumed a supersonic gas flow at large cometocentric distances and a subsonic flow close to the nucleus; in this case there is one and only one transonic solution which passes smoothly through the sonic point. Transonic solutions can not, in principle, be obtained numerically without additional assumptions. Generally, it has been assumed that the gas velocity (or Mach number) and its first derivative behaved continuously at the sonic point. Following Probstein's original work $3 /$, practically all early transonic coma calculations used a type of "shooting method": the initial flow velocity (or Mach number) value was "fine tuned" until a transonic solution was reached $/ 6,8,9,10,13 /$. In order to avoid the numerical difficulties of the earlier treatments and also to make it possible to describe dynamic phenomena in the coma, the first time-dependent (but still spherically symmetric) dusty gas dynamic model of the gas-dust interaction region was developed by Gombosi et al. $\pi /$. The method was later combined with a three-dimensional, kinetic dust treatment in the outer coma $/ 14 \%$. In this model the gas dynamic equations are solved using a modified version of Godunov's first scheme which can naturally handle shocks and discontinuity surfaces. The model was able to describe such phenomena as dust halo formation in the inner coma, temporal evolution of dust and gas parameters following a comet outburst, etc. However, the numerical model can still use further improvements. In its first published form the model assumed a rather simple photochemistry, gas collisional cooling, neglected infrared radiation trapping $/ 2 /$, and assumed that the total radiative energy flux was approximately constant everywhere in the coma (an assumption that is more or less justified on the basis of earlier radiative transfer calculations $/ 10,15 /)$.

\section{TIME-DEPENDENT, MULTIDIMENSIONAL DUSTY JET MODELS}

The comet Halley imaging experiments showed that cometary activity is concentrated to limited areas on the sunlit side of the nucleus, with most of the dust ejection coming from fairly localized jets. Spherically symmetric, steady-state models were proven to be totally inadequate to describe cometary inner regions. A few years ago Kitamura has developed the first time-dependent 2D dusty gas-dynamic code using one characteristic dust size, simple energetics, and a highly simplified chemistry $/ 11 /$ Kitamura's model $/ 11 /$ has problems with the time-dependent treatment of the gas outflow, therefore he published only steady-state results. Nevertheless, his first 2D calculation represents an important step forward in describing dusty jets. Fig.2 shows steady-state gas and dust density distributions obtained with his $2 \mathrm{D}$ axially symmetric model $/ 11 /$. The nucleus represents an isotropic background source with a Gaussian shaped subsolar active region having a characteristic half opening angle of $10^{\circ}$. The gas and dust production rates are a factor of 10 higher inside the active region than outside. In Kitamura's first model /11/ the gas density at the surface varies as 


$$
\rho(\theta)=\rho_{0}\left[(\alpha-1) \exp \left(-\frac{\theta^{2}}{\theta_{0}^{2}}\right)+1\right]
$$

Here $\alpha=10$ and $\theta_{0}=10^{\circ}$. It should be noted that in this model the contribution of the jet to the total gas and dust production is very small: the ratio of jet production to the background production is only about 0.07 , therefore most of the gas anid dust is released outside the jet. Inspection of Fig. 2 reveals that the originally $10^{\circ}$ wide active region eventually produces a much broader jet (the effective half opening angle is about $35^{\circ}$ ). The physical reason for this broadening is that the horizontal pressure gradient generates lateral gas flow, which in turn accelerates the dust grains in the horizontal direction, too. This process in effect "sweeps out" most of the grains from the subsolar region, i.e., from the region above the active region. The horizontal expansion represents an additional expansion direction for the gas released from the active region, therefore, the pressure of this gas component decreases faster than that of in the background gas. At some point there will be a pressure balance between the gas in the jet and in the background: this balance eventually stops the horizontal gas expansion. This means that there will be a dust accumulation near the pressure balance surface, which in this case happens to be a conical surface with a half opening angle of about $35^{\circ}$. Kitamura's result is very interesting: it indicates that weak jets may eventually cause a dust density decrease above the active area and that the dust accumulation is concentrated to a conical surface much beyond the angular extent of the jet itself.

In a follow-up paper, Kitamura investigated the case of isolated jets, i.e., when all gas and dust production is concentrated to a localized subsolar active area $/ 12 /$. In this paper the governing equations were solved only for the dayside (there was a low pressure external boundary at $\theta=90^{\circ}$ ). Also, at the nuclear surface outside the active area Kitamura /12/ adopted slip flow conditions to describe the interaction of the gas flow with the nucleus. It is our opinion that the introduction of a low pressure boundary at $\Theta=90^{\circ}$ is highly questionable, because it results in significant gas flows through the terminator at all cometocentric distances even under steady-state conditions. Physically this boundary condition is equivalent to a very strong "vacuum cleaner" placed to the nightside. As it will be shown later in this paper our calculations (which solve the coupled axisymmetric gas and dust equations for both the dayside and the nightside) indicate that there is a very significant day to night flow close to the nucleus, but this transterminator flow practically disappears beyond about $2 \mathrm{R}_{\mathrm{N}}$. In the same paper, Kitamura $/ 12 /$ also investigated an other situation, which is more consistent with his numerical model. In this case the gas production was assumed to be uniform all over the nucleus (no cross terminator flow), while the dust production was concentrated to a limited subsolar area. Fig. 2. shows the gas and dust density distributions obtained with this model $/ 12 /$. It is obvious from the description of the physical problem that horizontal gas pressure gradients will develop only as a result of the dust mass loading, therefore one can only expect moderate horizontal gas and dust transport Inspection of Fig. 3.shows that this is really the case. One can clearly see the effects of the gas-dust interaction in the subsolar region. One could say that this solution is essentially a combination of a spherically symmetric radial dusty gas flow in the subsolar region and a pure spherically symmetric radial gas flow elsewhere.

Very recently Gombosi and Körösmezey developed a new 2D, time-dependent, axisymmetric dusty gas dynamic jet model $/ 18,31$. The model solves the coupled, time-dependent continuity, momentum and energy equations for a dust-gas mixture (equations (1) through (11)). The partial differential equation system was solved using a second order accurate upwind biased Godunov type scheme with time-splitting recently developed at the University of Michigan. This technique is applicable to initial value problems governed by hyperbolic partial differential equations. The details of the new numerical technique will published elsewhere $31 \%$. The equations were solved on $40 \times 40$ mesh. A linearly spaced grid structure was adopted in the azimuthal direction (extending from $0^{\circ}$ to $180^{\circ}$ ) while the logarithmically spaced radial grids extended from 6 to $100 \mathrm{~km}$.

\section{Gas density}

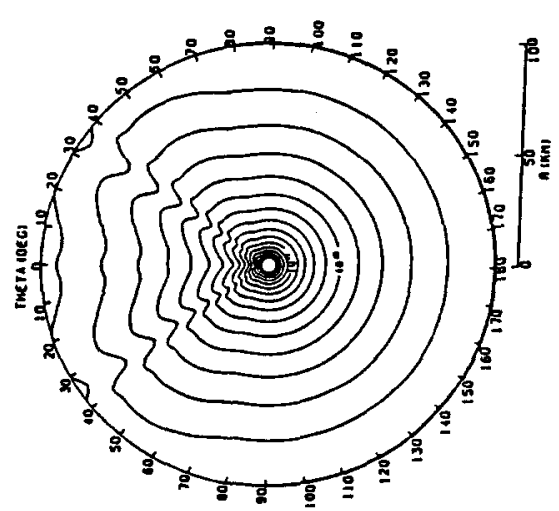

Dust density

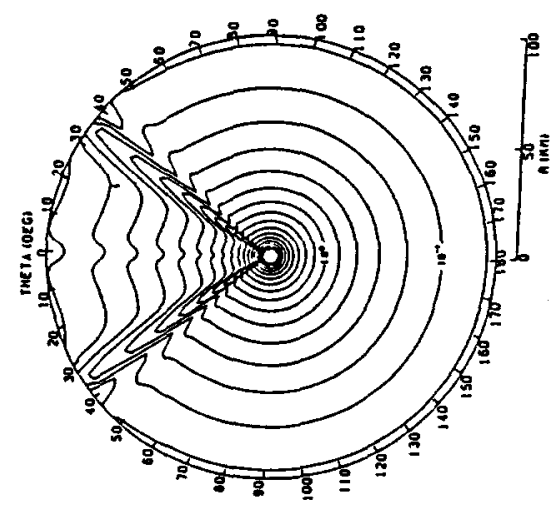

Fig. 2. Gas and dust isodensity contours in the inner cometary coma. The nucleus is modeled as an isotropic background source of gas and dust with a narrow subsolar active region. The jet/background production rate ratio is 

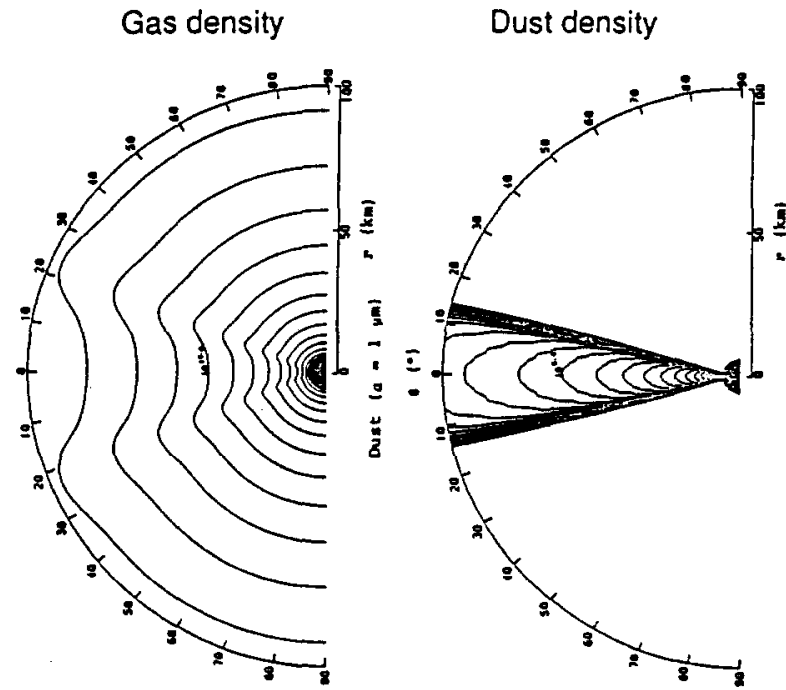

Fig. 3. Gas and dust isodensity contours in the inner cometary coma in the cas of an isolated dust jet. The nucleus is considered to be a uniform source of cometary gas, while dust production is concentrated to a narrow subsolar active region only. (from $/ 12 /$ ).

It is assumed that initially $(t=0)$ the near nucleus region is dust free and filled with low density gas. At $t=0$ an active area was generated on the nucleus surface $\left(R_{n}=6 \mathrm{~km}\right)$. The gas production was self-consistently calculated at every time step using the reservoir outflow boundary condition of Gombosi et al. $n /$. The sublimation reservoir density was obtained as a surn of three terms:

$$
\rho\left(R_{N}, \theta, t\right)=\rho_{\text {jet }}(\Theta, t)+\rho_{\text {bckg }}(\Theta, t)+\rho_{\text {init }}
$$

where $\rho_{\text {init }}=3 \times 10^{-13} \mathrm{~g} / \mathrm{cm}^{3}$ represents a very weak initial gas source (active before $t=0$ ), $\rho_{\text {bckg }}$ is an anisotropic background source, $\rho_{b c k g}=\rho_{0} \cos \theta$ if $\Theta<85^{\circ}$ and $\rho_{b c k g}=0.1 \rho_{0}$ if $\theta>85^{\circ} \quad\left(\rho_{0}=2.5 \times 10^{-9} \mathrm{~g} / \mathrm{cm}^{3}\right)$, while $\rho_{\text {jet }}$ describes the jet source in the subsolar active region.

$$
\rho_{\mathrm{jet}}=\rho_{0} \alpha \exp \left(-\frac{\Theta^{2}}{\theta_{0}^{2}}\right)
$$

Here $\mathrm{a} \theta_{0}=10^{\circ}$ value was adopted. The jet strength parameter, $\alpha$, was a free parameter of the model. In the present set of calculations $\alpha=15$ (weak jet) and $\alpha=75$ (strong jet) values were used. At the beginning of the calculation the jet density was increased to full strength with an e-folding time scale of $100 \mathrm{~s}$. The sublimation temperature was $200 \mathrm{~K}$, while the surface dust temperature was $418 \mathrm{~K}$. For the sake of simplicity only one dust size is considered in the present model $(\mathrm{a}=0.65 \mu \mathrm{m})$. The dust production rate was self-consistently calculated in time step using the friable sponge model of Horanyi et al. $22 \%$. The friability parameter was chosen to yield a dust to gas mass production ratio of about 0.3 , close to the observed Comet Halley value. A very low pressure external "vacuum cleaner" was placed at a distance of $100 \mathrm{~km}$, which helped to ensure a supersonic flow in most of the integration region (the sonic point was located at about $100 \mathrm{~m}$ from the surface.

Fig. 4 shows snapshots of the gas and dust densities following the onset of a weak jet. In this case the jet/background production rate ratio is about 0.6 , much larger than the value used by Kitamura in his paper $/ 11 /$ (he had a jet/background ratio of 0.07 ). The snapshots present two-dimensional equidensity curves at $t=30 \mathrm{~s}, 60 \mathrm{~s}, 90 \mathrm{~s}$, $166.7 \mathrm{~s}, 333.3 \mathrm{~s}$ and $500 \mathrm{~s}$ after onset. Inspection of Fig. 4 reveals a very interesting feature. Initially the newly ejected gas and dust expands practically radially with relatively little horizontal transport. The main reason for this almost entirely radial outflow pattem is that due to the $-10^{\circ}$ spatial extent of the source regior. at the nucleus (the active "spot" on the surface has a radius of about $1 \mathrm{~km}$ ) the surface radial pressure gradient is much larger than the azimuthal gradient. As the gas leaves the nucleus - coma interface region the azimuthal pressure gradient becomes comparable to the radial one and the gas flow starts to expand in the azimuthal direction as well. In this phase the gas expansion goes faster than $\mathrm{r}^{2}$ (especially near the outer edge of the jet), therefore two interesting things happen. First of all the jet cone expands to the nightside and secondly the dust grains also attain a significant azimuthal velocity component. A natural consequence of this azimuthal velocity component is that a large fraction of the dust particles is "swept" away from the region above the active area, thus resulting in a dust density depletion near the edges of the original jet. At the same time there is only a relatively weak horizontal transport near the axis of symmetry $\left(\Theta=0^{\circ}\right)$, therefore, the central region of the jet is practically not depleted. The end result is a dust density peak above the center of the active region (this peak was not obtained in Kitamura's solution /11/) and a secondary dust accumulation on the nightside, where the expanding gas reaches pressure balance with the background component. This nighttime accumulation of dust grains might help us to explain Giotto imaging observations indicating the presence of dust in the nightside of comet Halley $/ 16 \%$. 


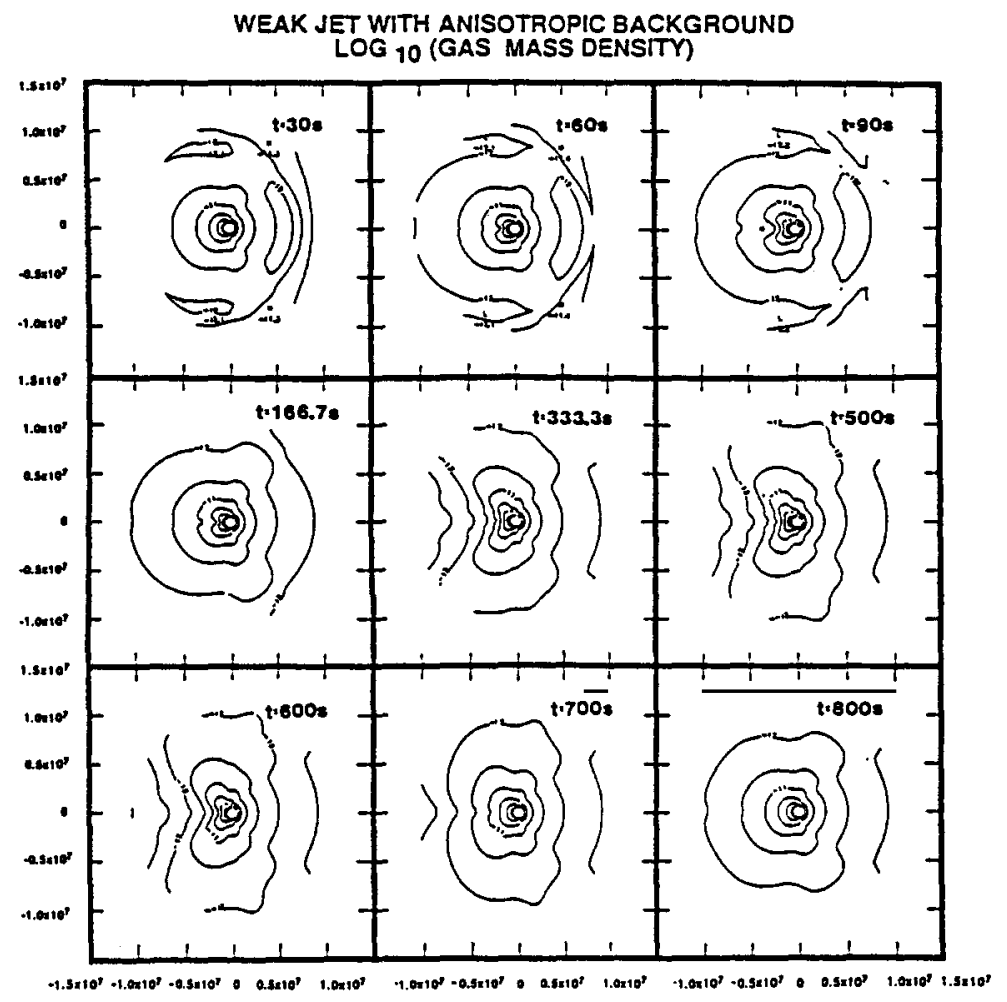

Fig. 4.a. Snapshots of gas isodensity contours following the onset of a weak jet at $t=0$. The panels show the gas density contours at $t=30 \mathrm{~s}, 60 \mathrm{~s}, 90 \mathrm{~s}, 166.7 \mathrm{~s}, 333.3 \mathrm{~s}$ and $500 \mathrm{~s}$. In each panel the axis of symmetry is a horizontal line going through the center. The sunward direction is to the left of each panel.

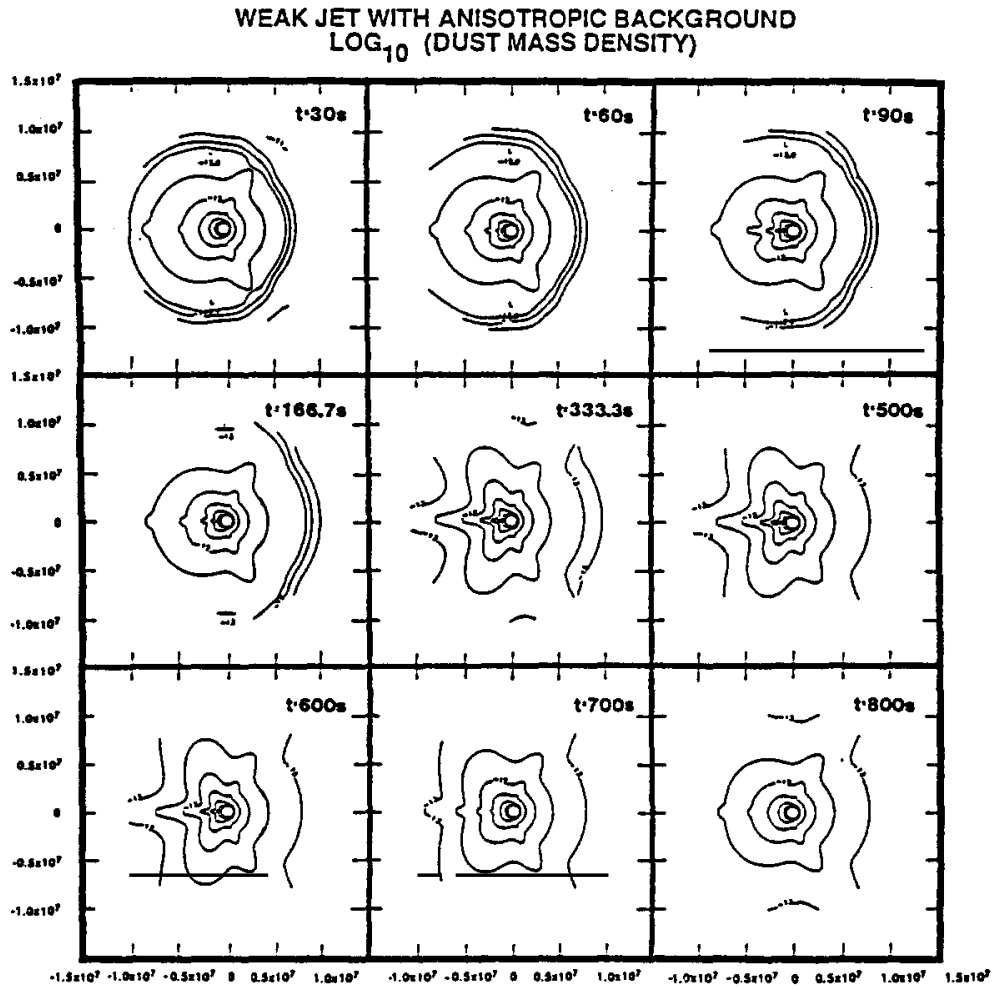

Fig. 4.b. Snapshots of dust isodensity contours following the onset of a weak jet at $t=0$. The panels show the gas density contours at $t=30 \mathrm{~s}, 60 \mathrm{~s}, 90 \mathrm{~s}, 166.7 \mathrm{~s}, 333.3 \mathrm{~s}$ and $500 \mathrm{~s}$. In each panel the axis of symmetry is a horizontal line going through the center. The sunward direction is to the left of each panel. 
By about $t=100$ s the azimuthal gas expansion is stopped by the background gas. It has already been demonstrated by Kitamura's first calculation $/ 11 /$ that this effect is quite sensitive to the background gas production rate (gas production from the inactive part of the nucleus). This means that beyond about $50 \mathrm{~km}$ or so there is no significant azimuthal gas velocity and consequently the dust particles also lose most of their azimuthal velocity component. The late time (few hundred seconds) distributions, which exhibit similar general features as Kitamura's steady-state results $/ 11 /$, show that most of the dust is concentrated to a very narrow subsolar jet and to a conical surface with half-opening angle of

Fig. 5 shows snapshots of the gas and dust densities following the onset of a strong jet. In this case the jet/background production rate ratio is about 3 . The snapshots present two-dimensional equidensity curves at $t=30 \mathrm{~s}$, $60 \mathrm{~s}, 90 \mathrm{~s}, 133.3 \mathrm{~s}, 266.7 \mathrm{~s}$ and $400 \mathrm{~s}$ after onset. The general features of the solution are very similar to those obtained in the case of a weak jet. The main difference is that in the case of the strong jet the subsolar dust peak and the nighttime dust cone are much more pronounced than they were in the weak jet case. The opening angle of the dust and gas cone is about $150^{\circ}$, larger than it was in the previous case The reason is now the subsolar pressure in the active region is larger, therefore the pressure balance with the background gas will be achieved at a larger solar zenith angle.

Our conclusion is that the structure of a dusty jet resulting from an axisymmetric active region is cone-like with a very narrow, subsolar spike. The opening angle of the dust cone is largely determined by the ratio of the gas pressures inside the and outside active surface area. This jet structure is significantly different from the ones predicted by earlier spherically symmetric calculations (cf. $25,28 /$ ), Our results are in a good qualitativ agreement with the results of Kitamura /11/ with the exception of the subsolar spike which was not predicted earlier.

STRONG JET WITH ANISOTROPIC BACKGROUND LOG $_{10}$ (GAS MASS DENSITY)

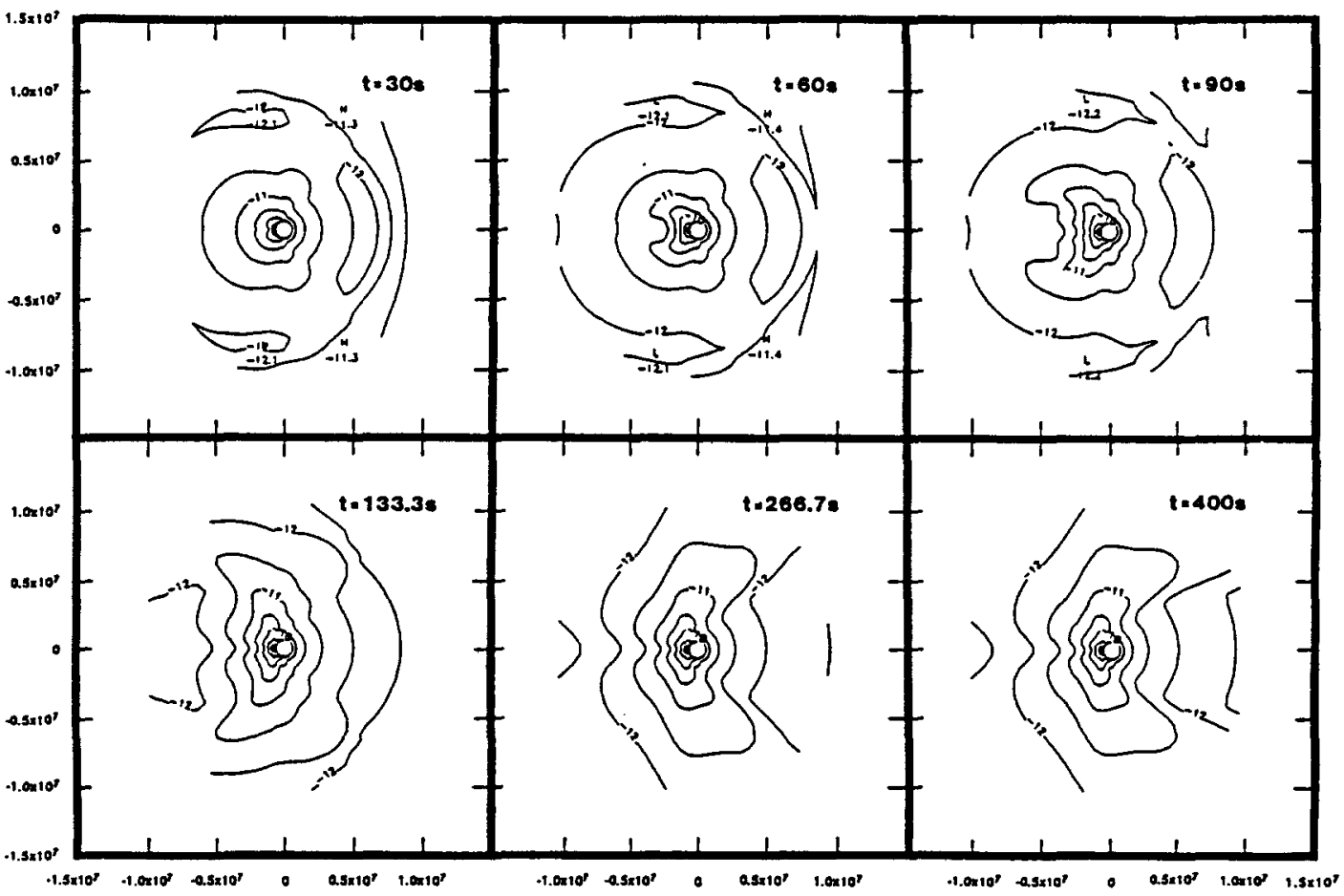

Fig. 5.a. Snapshots of gas isodensity contours following the onset of a strong jet at $t=0$. The panels show the gas density contours at $t=30 \mathrm{~s}, 60 \mathrm{~s}, 90 \mathrm{~s}, 133.3 \mathrm{~s}, 266.7 \mathrm{~s}$ and $400 \mathrm{~s}$. In each panel the axis of symmetry is a horizontal line going through the center. The sunward direction is to the left of each panel. 


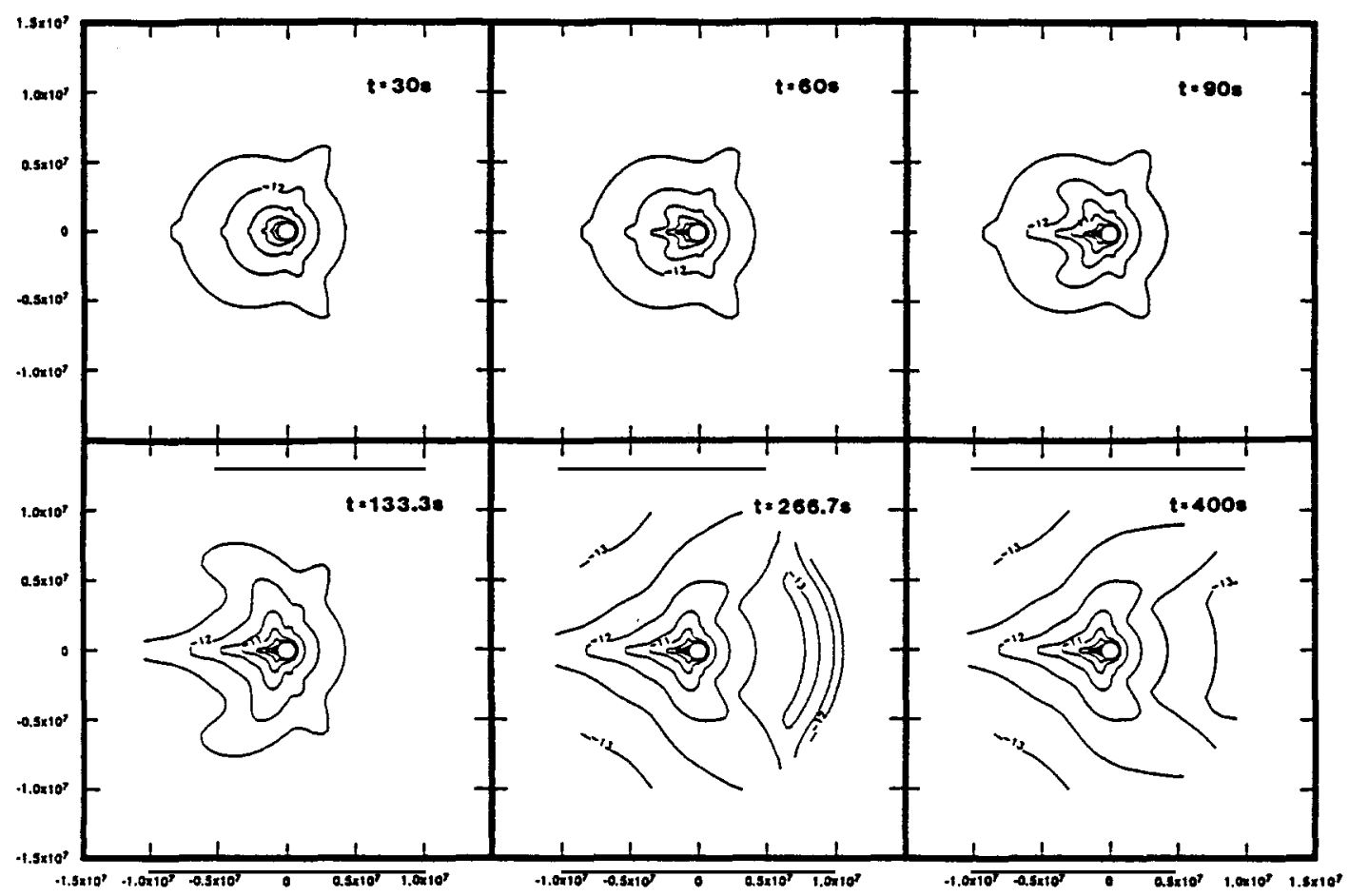

Fig. 5.b. Snapshots of dust isodensity contours following the onset of a strong jet at $\mathrm{t}=0$. The panels show the gas density contours at $t=30 \mathrm{~s}, 60 \mathrm{~s}, 90 \mathrm{~s}, 133.3 \mathrm{~s}, 266.7 \mathrm{~s}$ and $400 \mathrm{~s}$. In each panel the axis of symmetry is a horizontal line going through the center. The sunward direction is to the left of each panel.

\section{ACKNOWLEDGEMENTS}

The authors are indebted to Dr.Bram van Leer for his advice in developing the second order upwind biased Godunov type numerical scheme to solve the coupled, time-dependent multidimensional partial differential equation system. This work was supported by NSF grant AST-8605994, and NASA grant NAGW-1366. Acknowledgement is also made to the National Center for Atmospheric Research sponsored by NSF, for the computing time used in this

\section{REFERENCES}

1. F.L. Whipple, A comet model I., The acceleration of comet Encke, Astrophys. Joumal, 111, 375 (1950)

2. M.L. Marconi, and D.A. Mendis, IR heating of Comet Halley's atmosphere, Earth. Moon and Planets, 36, 249 (1986)

3. R.F. Probstein, The dusty gas dynamics of comet heads, in: Problems of Hydrodynamics and Continuum Mechanics, published by the Soc. Industr. Appl. Math., p568 (1968)

4. Shulman, L.M., Dinamika Kometnukh A tmospher - Neutral'nvi Gaz, Naukova Dumka, Kiev, 1972.

5. Hellmich, R., and H.U. Keller, On the dust production rates of comets, in Solid Particles in the Solar System (eds. J. Halliday and B.A. McIntosh), p255, D. Reidel, Hingham, Mess., 1980.

6. T.I. Gombosi, K. Szegö, B.E. Gribov, R.Z. Sagdeev, V.D. Shapiro, V.I. Shevchenko, T.E. Cravens, Gas dynamic calculations of dust terminal velocities with realistic dust size distributions, Cometary Explomation (ed. T. I. Gombosi), KFKI Press, Budapest, Hungary, Vol 1., 99, 1983.

7. T.I. Gombosi, T.E. Cravens, and A.F. Nagy: Time dependent dusty gas dynamical flow near cometary nuclei, Astrophysical Journal, 293, 328, (1985)

8. Marconi, M.L., and Mendis D.A., The photochemical heating of the cometary atmosphere, Astrophys. Ioumal, 260, 386, (1982)

9. M.L. Marconi, and D.A. Mendis, The atmosphere of a dirty cometary nucleus, A two-phase multi-fluid model, Astrophys. Journal, 273, 381, (1983)

10. M.L. Marconi, and D.A. Mendis, The effects of the diffuse radiation fields due to multiple scattering and thermal reradiation by dust on the dynamics and thermodynamics of a dusty cometary atmosphere, Asmophus Loumal, 287, 445, (1984)

11. Y. Kitamura, Axisymmetric dusty gas jet in the inner coma of a comet, Icarus, 66, 241, (1986)

12. Y. Kitamura, Axisymmetric dusty gas jet in the inner coma of a comet II. The case of isolated jets, Icarus, 72, 555, (1987)

13. R. Hellmich, Anisotrope Mehrfachstreunung in der Staubkoma und ihr Einfluss auf die Sublimationsrate des Kometenkems, PhD thesis, Univ. Görtingen, FRG, 1979. 
14. T.I. Gombosi, and M. Horányi, Time-dependent modeling of dust halo formation at comets, Astrophysical Joumal, 311, 491, (1986)

15. H.U. Keller, Dust and gas models in the coma, in Cometary Exploration (ed. T.I. Gombosi), Vol. 1, p119, Hungarian Academy of Sciences, Budapest, 1983.

16. H.U. Keller, private communication (1988)

17. N.I. Kömle and W.-H. Ip, Anisotropic non-stationary gas flow dynamics in the coma comet P/Halley, Astron. Astrophys., 187, 405 (1987)

18. T.I. Gombosi and Körösmezey A., Cometary dusty gas dynamics, in: Outstanding Problems in Solar Svstem Plasma Physics eds. J. Burch and H. Waite, AGU, in press (1988)

19. P.R. Weissman and H.H. Kieffer, Thermal modeling of cometary nuclei, Icanus, 47, 302, (1981)

20. D.A. Mendis, and G.D. Brin, On the monochromatic brightness variations of comets II, The core-mantle model, Moon and Planets, 17, 359, (1977)

21. G.D. Brin, and D.A. Mendis, Dust release and mantle development in comets, Astrophys. J, 229, 402, (1979)

22. M. Horányi, T.I. Gombosi, T.E. Cravens, A. Körösmezey, K. Kecskeméty, A.F. Nagy, K. Szegö: The friable sponge model of a cometary nucleus, Astrophysical Journal, 278, 449, (1984)

23. F.P. Fanale, and J.R. Salvail, An idealized short-period comet model: Surface insolation, $\mathrm{H}_{2} \mathrm{O}$ flux,dust flux and mantle evolution, Icarus, 60, 476, (1984)

24. H.L.F. Houpis, W.-H. Ip, and D.A. Mendis, The chemical differentiation of the cometary nucleus: The process and its consequences, Astrophys. Joumal. $295654,(1985)$

25. T.I. Gombosi, A.F. Nagy, and T.E. Cravens: Dust and neutral gas modeling of the inner atmospheres of comets, Reviews of Geophysics, 24, 667, (1986)

26. A.H. Delsemme and P. Swings, Hydrates de gaz dans les noyaux cometaires et les grains interstellaires, Ann. Astrophys. 15, 1, (1952)

27. E. Kürth, D. Möhlmann, B. Giese, and F. Tauber, Thermal stresses and dust dynamics on comets, in Proc. 20th ESLAB Symposium on the Exploration of Halley's Comet (eds. B. Battrik, E.J. Rolfe and R. Reinhard), ESA SP-250, Vol. 2, p385, 1986.

28. D.A. Mendis, H.L.F. Houpis and M.L. Marconi, The Physics of Comets, Fundamentals of Cosmic Physics, $10,1,(1985)$

29. M. Shimizu, Neutral temperature of cometary atmospheres, in: The Study of Comets (eds.B. Donn, M. Mumma, W. Jackson, and R. Harrington), NASA S-393, p363, 1976.

30. J. Crovisier, The water molecule in comets: fluorescence mechanisms and thermodynamics of the inner coma, Astronomv and Astrophysics, 130, 361, 1984.

31. A. Körösmezey and T.I. Gombosi, A time-dependent dusty-gasdynamic model of axisymmetric cometary jets, in preperation (1988) 